\title{
Magnetic Properties of the Superconductor $\mathrm{LaCaBaCu}_{3} \mathrm{O}_{7}$
}

\author{
Luis De Los Santos V., ${ }^{*}, 2$, Angel Bustamante D. ${ }^{3}$, J.C. Gonzalez ${ }^{4}$, Juan Feijoo L. ${ }^{3}$, Ana Osorio A. , \\ Thanos Mitrelias $^{1}$, Yutaka Majima ${ }^{2}$ and Crispin H.W. Barnes ${ }^{1}$
}

\author{
${ }^{1}$ Cavendish Laboratory, University of Cambridge, J.J Thomson Av. Cambridge CB3 OHE, United Kingdom \\ ${ }^{2}$ Materials and Structures Laboratory, Tokyo Institute of Technology, 4259 Nagatsuta-cho, Yokohama 226-8503, Japan \\ ${ }^{3}$ Lab. Superconductividad, Facultad de Ciencias Físicas, Universidad Nacional Mayor de San Marcos, Ap. Postal 14- \\ 0149. Lima, Perú \\ ${ }^{4}$ Instituto de Ciencia de Materiales de Sevilla - CSIC, Av. Américo Vespucio 49, Sevilla 41092, Cádiz - España \\ ${ }^{5}$ Facultad de Química e Ingeniería Química, Universidad Nacional Mayor de San Marcos, Av. Venezuela S/N, Lima 1, \\ Perú
}

\begin{abstract}
LaCaBaCu}_{3} \mathrm{O}_{7}$ (La1113) is a superconductor below $T_{\mathrm{C}(\text { onset })}=80 \mathrm{~K}$ and its structure is similar to the tetragonal YBCO. In this work the magnetic properties of a bulk La1113 sample are reported and compared to those of YBCO superconductor. We explore a possibly relation of this property to its particular crystalline structure, particularly to the oxygen deficiency. In contrast to a bulk YBCO, some magnetic characteristics of La1113 below $T_{\mathrm{C}}$ are: the magnetic flux can penetrate easily the bulk in its vortex region $\left(H_{\mathrm{C} 1}<H<H_{\mathrm{C} 2}\right)$, fewer fields can be trapped than in YBCO, small currents are able to flow in the bulk and possible applications of La1113 are restricted under applied fields lower than 10KOe and in the range of $41 \mathrm{~K}-76 \mathrm{~K}$. The critical current density is determined by currents flowing through grain boundaries. Peak effect is observed and it is probably caused by secondary phases or from clusters of oxygen vacancies acting as field induced pins. A first magnetic phase diagram for La1113 showing the irreversibility line is reported here. Finally, we found that the specific heat capacity of La1 113 is $37.2 \mathrm{~mJ} / \mathrm{K}^{2} \mathrm{~mol}$. C 2010 . All rights reserved
\end{abstract}

Keywords: Cuprates superconductors, general properties of high-Tc superconductors, magnetic properties of superconductors, critical currents of superconductors, critical fields of superconductors, crystal structure of superconductors. PACS: 74.72.-h; 74.78.Bz; 74.25.-q; 74.25.Ha; 74.25.Sv; 74.25.Op; 74.62. Bf.

\section{INTRODUCTION}

It has been reported that slight variations in the crystalline structure of a high temperature cuprate superconductor (HTCS) produce large changes in its magnetic properties [1]. Structural variations are usually produced by chemical substitutions of cations or anions. Substitutions result in chemical pressure in some places of the structure thus altering the position and occupancy of the atoms situated in the charge reservoirs or in the superconducting planes $\mathrm{CuO}_{2}$, and also increasing (or decreasing) the superconducting critical temperature $\left(T_{\mathrm{C}}\right)[2,3]$. Another common method to modify the structure of a HTCS is by changing the vacancy of the oxygen atoms in the charge reservoirs. For instance, it is well known that by changing the oxygen content of the superconductor $\mathrm{YBa}_{2} \mathrm{Cu}_{3} \mathrm{O}_{7}$ (YBCO-7) to less than 6.4, the occupancy of the oxygen situated in the $\mathrm{Cu}-\mathrm{O}$ chains decreases and the crystalline structure changes from orthorhombic to tetragonal whit the superconductivity destroyed (see Figs. 1a and 1b). Another example is the peak effect which appears on critical current density $\left(J_{\mathrm{C}}\right)$ plots of structurally

*Address correspondence to this author at the Cavendish Laboratory, University of Cambridge, J.J Thomson Av. Cambridge CB3 0HE, United Kingdom; Tel: +44-1223-337006; Fax: +44-1223-337200;

E-mails: 1d301@cam.ac.uk, luisitodv@yahoo.es altered HTCSs [4,5]. This can originate from structural defects such as intrinsic pinning of vortices at the $\mathrm{CuO}_{2}$ planes [6] or from oxygen vacancy clusters [7, 8]. Most studies about altered HTCS structures have been focused on YBCO [9]. However, another interesting HTCS which could help us to find any possible relationship between structural and magnetic properties is $\mathrm{LaCaBaCu}_{3} \mathrm{O}_{7}$ ( $\mathrm{La1113}$ ). The two main characteristics which make La1113 attractive are (i) that its $T_{\mathrm{C}}$ is above the boiling temperature of liquid nitrogen $\left(T_{\mathrm{C}} \approx 80 \mathrm{~K}[10-14]\right)$ and (ii) that its structure is tetragonal similar to the non-superconductor $\mathrm{YBa}_{2} \mathrm{Cu}_{3} \mathrm{O}_{6}$ (YBCO-6) [15-17]. Fig. (1) shows schematically a structural comparison of La1113 with YBCO: YBCO-7 has an orthorhombic Pmmm structure and becomes superconductor below $T_{\mathrm{C} \text { (onset) }}$ of around $90 \mathrm{~K}$ (Fig. 1a), YBCO-6 has tetragonal $\mathrm{P} 4 / \mathrm{mmm}$ structure and is antiferromagnetic insulator (Fig. 1b). La1113 is superconductor and has tetragonal structure similar to YBCO-6 (Fig. 1c). The literature reports indicate that this superconductor does not show any structural variations after single atom substitution. For instance, its tetragonal structure does not change under different oxygen content $[18,19]$ or when the $\mathrm{Cu}(1)$ site $((0,0,0)$ site in Fig. 1) is doped with $\mathrm{Zn}$ content [19, 20]. However, La1113 becomes orthorhombic (Pmmm) when polyhedrons formed by a cation and oxygen atoms such as oxyanions $\left(\mathrm{BO}_{3}\right)^{3-}$ or $\left(\mathrm{PO}_{4}\right)^{3-}$ are located in the $\mathrm{Cu}(1)$ site [21-23]. Whatever the 


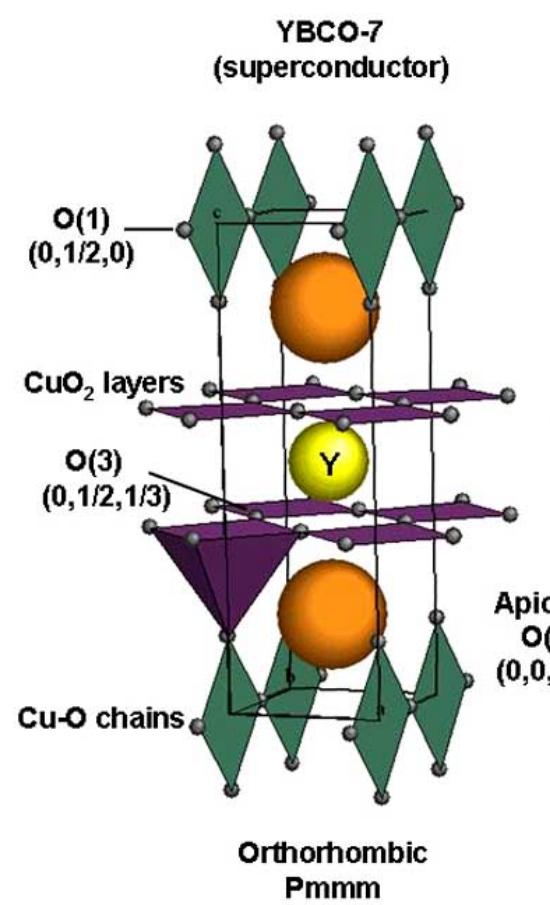

(a)

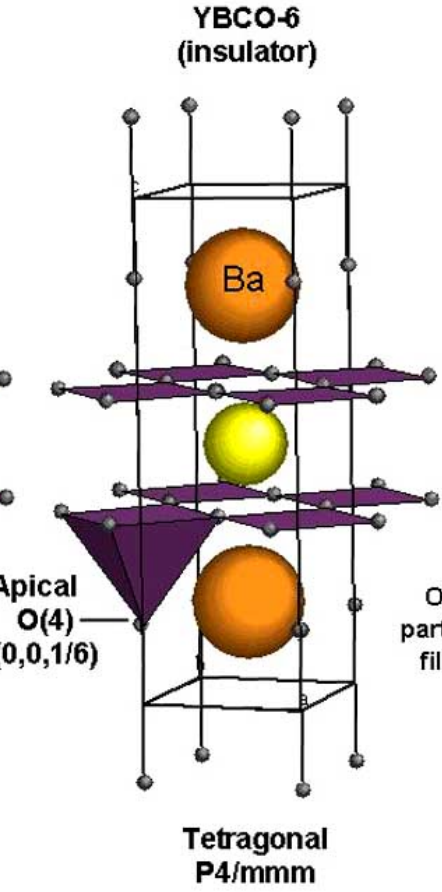

(b)

\section{La1113 \\ (superconductor)}




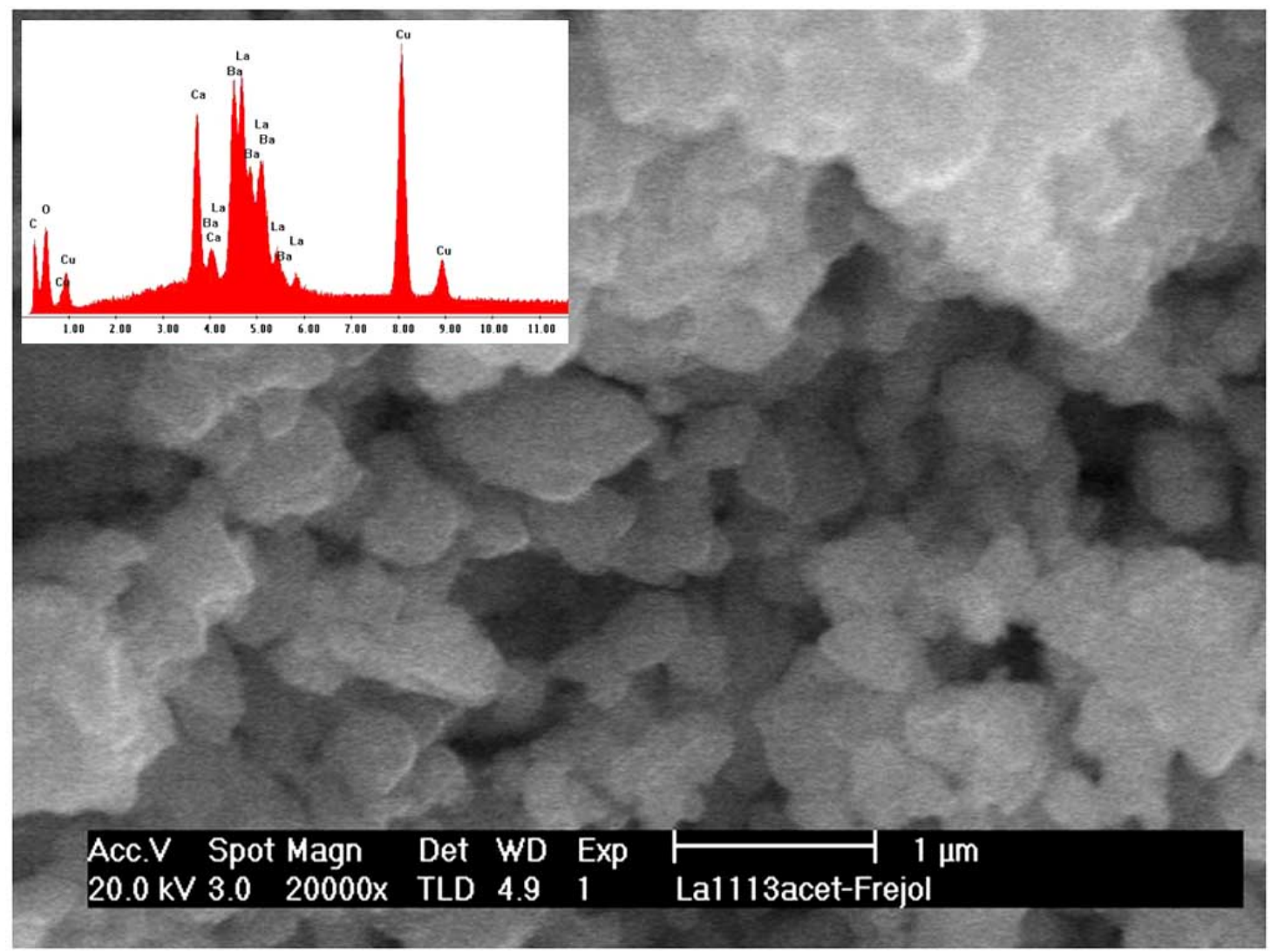

Fig. (2). Scanning electron micrograph of the superconductor $\mathrm{LaCaBaCu}_{3} \mathrm{O}_{7}$.

YBCO sample has a superconducting phase below $T_{\mathrm{C}(\text { onset) }}$ $\approx 90 \mathrm{~K}$.

\section{RESULTS AND DISCUSSION}

A scanning electron micrograph of the superconductor $\mathrm{LaCaBaCu}_{3} \mathrm{O}_{7}$ is presented in Fig. (2). A granular morphology is obtained following the precipitation technique described above. The grain sizes are in the $\mathrm{nm}$ scale and they are more uniform than those obtained following the solid state reaction method in which the shape and size of grains differ in tens of microns [21-23]. The mean crystallite diameter obtained from XRD analysis (not shown here) using the Scherrer's formula [27] is $73 \mathrm{~nm}$. The fact that nearly uniform grain sizes have been obtained after the preparation of the sample is important for the magnetic analysis. Non-regular grains implies grain boundaries with different areas and thus less uniform Josephson junctions between superconducting grains as we discuss below. The chemical composition analysis obtained by EDX over the whole area in the figure indicates the following molecular weights percentages: $28 \% \mathrm{La}, 28 \% \mathrm{Ba}, 7 \% \mathrm{Ca}, 19 \% \mathrm{Cu}$ and $18 \% \mathrm{O}$.

We now focus on studying the magnetic properties of the La1113 and any relationship with the sample's structural characteristics mentioned above. Since La1113 is a HTCS (Type II superconductor) some of its basic properties can be described by the field-dependence magnetization which also provides information about the trapped fields in it. The hysteresis loops of the bulk La1113 and YBCO sample at three different temperatures in the $\pm 20 \mathrm{kOe}$ range are shown in Fig. (3). The insets show the complete scan $( \pm 55 \mathrm{kOe})$ at $50 \mathrm{~K}$. It is noticeable from the figure that for both samples the magnetic moment for La1113 is twice that of YBCO (especially at low applied fields) and for any particular applied field $\left(H_{\mathrm{ext}}\right)$, the absolute value of the magnetization increases as the temperature decreases. For any of the temperatures shown in the figure, $H_{\mathrm{C} 1}$ (the lower critical field) of the La1113 loops (Fig. 3a) is closer to zero than for YBCO (Fig. 3b). This means that the Meissner state region $\left(H<H_{\mathrm{C} 1}\right)$ in La1113 is smaller than in YBCO, and the magnetic induction $(B)$ in the bulk La1113 can be reached at low applied fields. For both samples, the hysteresis loops become thin and close to linear when the temperature gets closer to their respective $T_{\mathrm{C}}$. Decreasing the temperature broadens the loops. Moreover, in the mixed state of La1113, between $H_{\mathrm{C} 1}$ and the upper critical field $H_{\mathrm{C} 2}$, La1113 has less magnetic moment than $\mathrm{YBCO}$ and decays to zero faster than the magnetization of YBCO. However in the negative $H_{\mathrm{C} 1}-$ $H_{\mathrm{C} 2}$ branches of the YBCO loops, the magnetic moment rapidly becomes negative. This behaviour is caused by the paramagnetic characteristic of its secondary phases. The presence of impurities or secondary phases in both samples is detected by analysing their magnetization curves as the temperature approaches $T_{\mathrm{C}}$. The $M(H)$ dependence for a clean and defect-free type II superconductor is reversible, and after switching off the external field, no magnetic flux is trapped within the superconductor. The $M(H)$ dependence becomes highly irreversible for superconductors containing defects or impurities which interact with the penetrating flux lines. The loops reveal irreversibility and remnant 


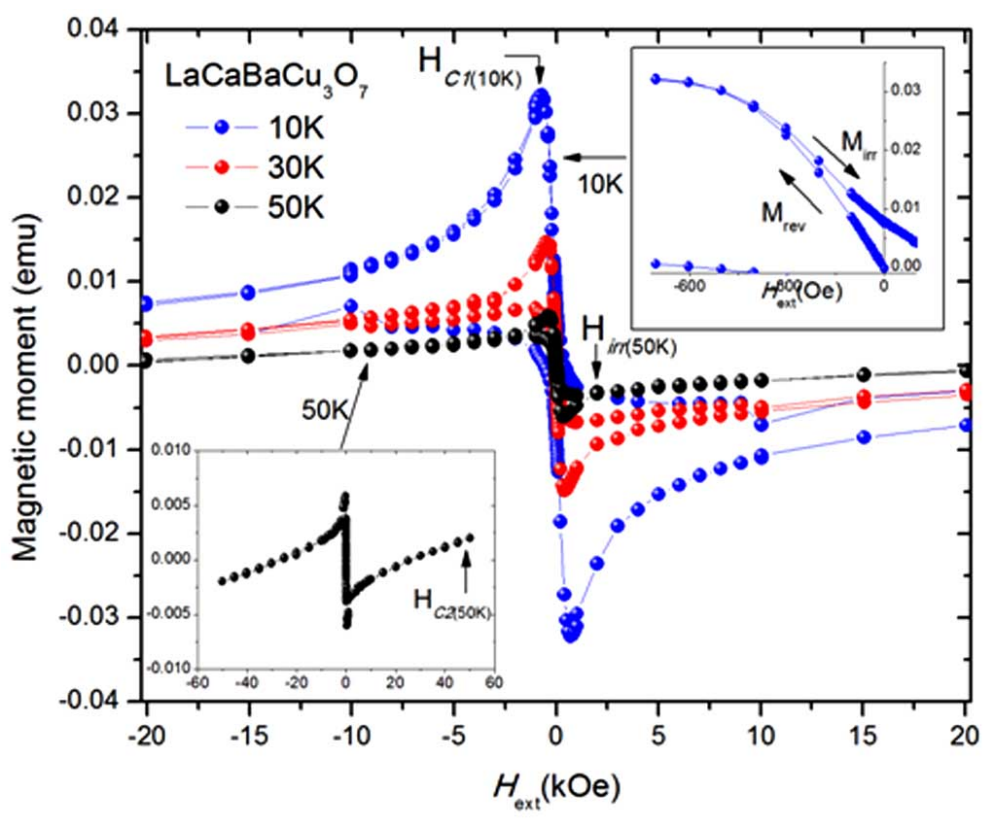

(a)

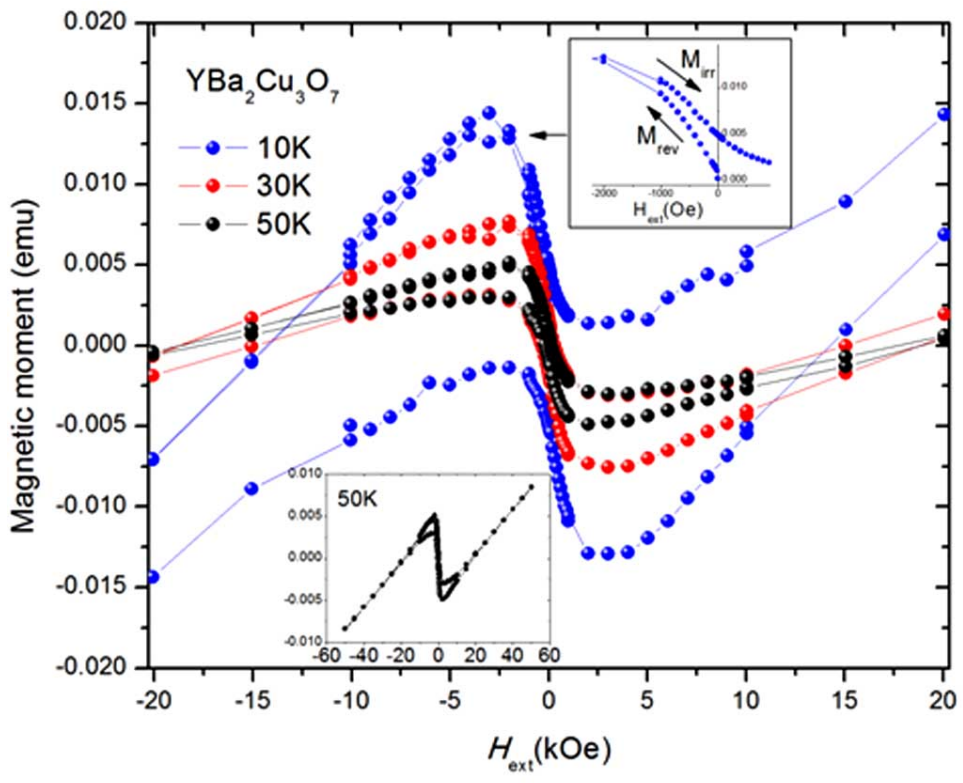

(b)

Fig. (3). Hysteresis loops of $\mathrm{LaCaBaCu}_{3} \mathrm{O}_{7}$ (a) and $\mathrm{YBCO}$ (b) at three different temperatures in their superconducting state.

magnetization at $10 \mathrm{~K}$ (see top insets in the figure) which remain at $50 \mathrm{~K}$. Secondary phases are commonly found after the preparation of HTCSs. The phases obtained for YBCO by the preparation technique used here are $\mathrm{Y}_{2} \mathrm{BaCuO}_{5}, \mathrm{CuO}$ and $\mathrm{BaCuO}_{2}$ [26], whereas for $\mathrm{La} 1113$ are $\mathrm{CaO}, \mathrm{Ca}_{2} \mathrm{CuO}_{3}$, $\mathrm{CuO}$ and $\mathrm{BaCuO}_{2}$ [11]. The presence of secondary phases is a possible explanation for the origin of the fishtail or peak effect [28, 29] as we will discuss below. Moreover, for both cases the magnetization $|M|$ decreases as the external field goes from $H_{\mathrm{C} 1}$ to $H_{\mathrm{C} 2}$. In the case of La1113, such decrease affects the critical current density values, the pinning force and could be caused by structural defects like partial occupancy of the oxygen in the $\mathrm{Cu}-\mathrm{O}$ chains in the structure as we will discuss next. The irreversible magnetization becomes zero at $H=H_{\text {irr }}$, with $H_{\text {irr }}$ as the irreversibility field, in contrast to the reversible magnetization which disappears at $H=H_{\mathrm{C} 2}$. Eventually, as the external field is reduced from $H_{\mathrm{C} 2}$ to $H_{\mathrm{C} 1}$ the magnetic moment becomes positive over a wide range of applied field indicating that not much magnetic field has been trapped in the La1113 superconductor as in YBCO. Therefore two characteristics can be observed from the hysteresis loops, the irreversibility and remnant magnetization are caused by presence of secondary phases whereas the decrease of magnetization under external field is possibly caused by structural defects.

The critical current density $J_{\mathrm{C}}$ for La1113 has been estimated from the irreversible $\mathrm{M}-\mathrm{H}$ loops at $70 \mathrm{~K}$ and $50 \mathrm{~K}$ 
using the Bean model [30] (approximation for grains superconductors with an average diameter $d[31]$ ):

$J_{C}=30 \frac{\Delta M}{d}$

Where $\Delta \mathrm{M}$ is the vertical width of the magnetization branches (forward $\mathrm{M}^{+}$minus downward $\mathrm{M}^{-}$) in $\mathrm{G}$ in the superconducting state, $d$ is the average diameter of the grains (in $\mathrm{cm}$ ). The obtained $J_{\mathrm{C}}$ for La1113 in $\mathrm{A} / \mathrm{cm}^{2}$ (shown in Fig. (4a)) exhibits strong field dependence with temperature and two different contributions to $J_{\mathrm{C}}$ are assumed. The "background" pinning represented by dashed lines in the figure is due to the La1113 bulk matrix. The critical current density of a bulk, polycrystalline HTCS material with randomly oriented grains is strongly limited by its pronounced weak link behavior. Only very small currents are able to flow across the grain boundaries when the external magnetic field is applied. This dependence is dominated by the classical 1/H law [32] reflecting the $J_{\mathrm{C}}(H)$ dependence of a percolation network of Josephson junctions ([33]).

In addition to the background pinning, the figure denotes an anomalous increase in the critical current density with magnetic field. At $70 \mathrm{~K}$ ( $6 \mathrm{~K}$ below the $T_{\mathrm{C}}$ ), a maximum $J_{\mathrm{C}}$ is observed near zero magnetic field followed by a peak from which the critical current density decreases monotonically from $8.6 \mathrm{kA} / \mathrm{cm}^{2}$ at $H \approx 1000$ to $2.3 \mathrm{kA} / \mathrm{cm}^{2}$ at $H_{\mathrm{ext}}=1 \mathrm{kOe}$. For $T=50 \mathrm{~K}$, the maximum $J_{\mathrm{C}}\left(\approx 90 \mathrm{kA} / \mathrm{cm}^{2}\right)$ is observed in the peak followed by a downward trend to higher values of $H_{\text {ext }}$. This effect is called "peak effect" and in the figure they are denoted as $H_{\mathrm{P} 70}$ and $H_{\mathrm{P} 50}$ respectively. We believe that the "peak effect" in the La1113 $J_{\mathrm{C}}$ plots is caused by "fieldinduced pins" (FIPs). The main contribution of FIPs might be clusters caused by the oxygen vacancies in the $\mathrm{Cu}-\mathrm{O}$ chains. These clusters, having a lower $\mathrm{H}_{\mathrm{C} 2}$ than the bulk La1113 matrix, represent weak pins and are disturbed at low applied fields [34]. The pins become stronger with increasing field because the superconducting order parameter in these disturbed regions would be much more strongly suppressed by the applied field than the order parameter of the whole bulk matrix. Hence, there is a certain range of magnetic fields, $20 \mathrm{Oe}-100 \mathrm{Oe}$ at $T=70 \mathrm{~K}$ and $0-200 \mathrm{Oe}$ at $T=50 \mathrm{~K}$, in which $\mathrm{J}_{\mathrm{C}}$ increases with increasing applied field before it starts to decrease above the fields $\mathrm{H}_{\mathrm{P} 70}$ and $\mathrm{H}_{\mathrm{p} 50}$ at which the $\mathrm{J}_{\mathrm{C}}$ peaks appear. Similar results have been obtained by altering the local distributions of the oxygen in the YBCO structure [7,8], where the authors concluded that the peak effect in YBCO is correlated with clustering of the oxygen vacancies. Like in the hysteresis loops measurements, the $J_{\mathrm{C}}$ plots are dominated by two contributions, the background contribution of inter-grain currents -typical of a bulk sample (represented by dashed lines in the figure) - and the intra-grain contribution of field-induced pinning (originating the peak effect) which might be caused by clusters of oxygen vacancies in the $\mathrm{Cu}-\mathrm{O}$ chains. The latest is better seen when the temperature is closer to $T_{\mathrm{C}}$.

The $J_{\mathrm{C}}$ data have also been used to obtain the volume pinning force $F_{\mathrm{P}}$ (force originated from interactions between the vortex lattice and pinning defects per volume) in the La1113 sample for $70 \mathrm{~K}$ and $50 \mathrm{~K}$ through the formula:

$$
F_{P}=J_{C} \times \mu_{0} H
$$

Fig. (4b) shows the normalized volume pinning force as a function of the reduced field $h=H_{\text {ext }} / H_{\text {irr. }} . H_{\text {irr }}$ is the natural boundary for flux pinning in the La1113 superconductor as it is shown below. For YBCO, $h \approx 0.33$ [35], this is represented by a solid blue line in the figure. According to [36], such value of $h$ is in accordance with pinning provided by normally-conducting or insulator regions $(\delta l$ pinning which is due to the scattering in the electron mean free path [37]). In the figure, the pinning force for La1113 is 0 at $H_{\mathrm{ext}}=0$ and $H_{\text {ext }}=H_{\text {irr }}$. The reduced field $h$ has values of 0.3 at $70 \mathrm{~K}$ and 0.33 at $50 \mathrm{~K}$. These values are closer to that for YBCO, indicating that the pinning mechanism in La1113 might be similar to that of YBCO at applied fields lower than $H_{\text {irr }}$.

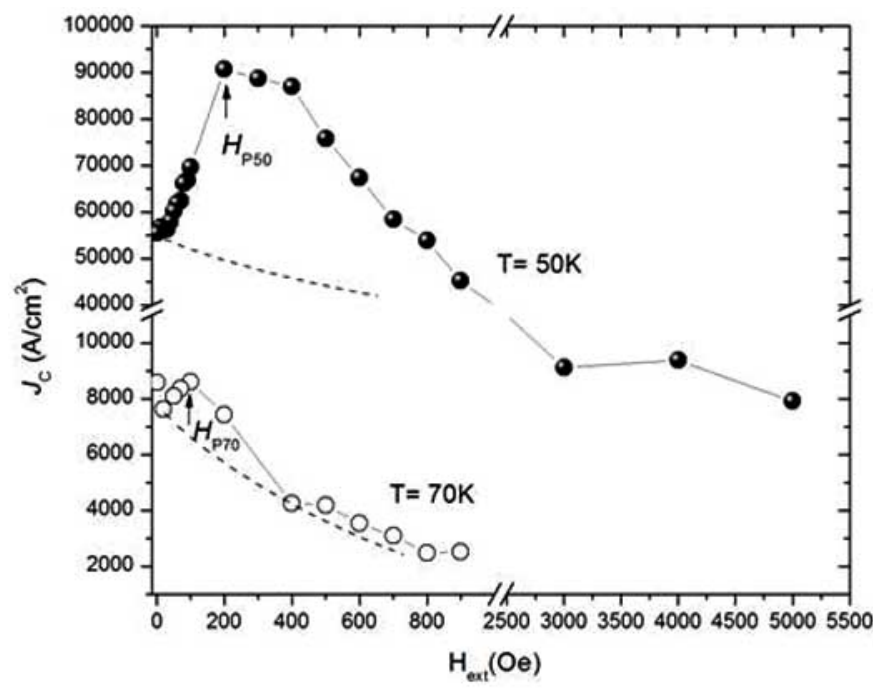

(a)

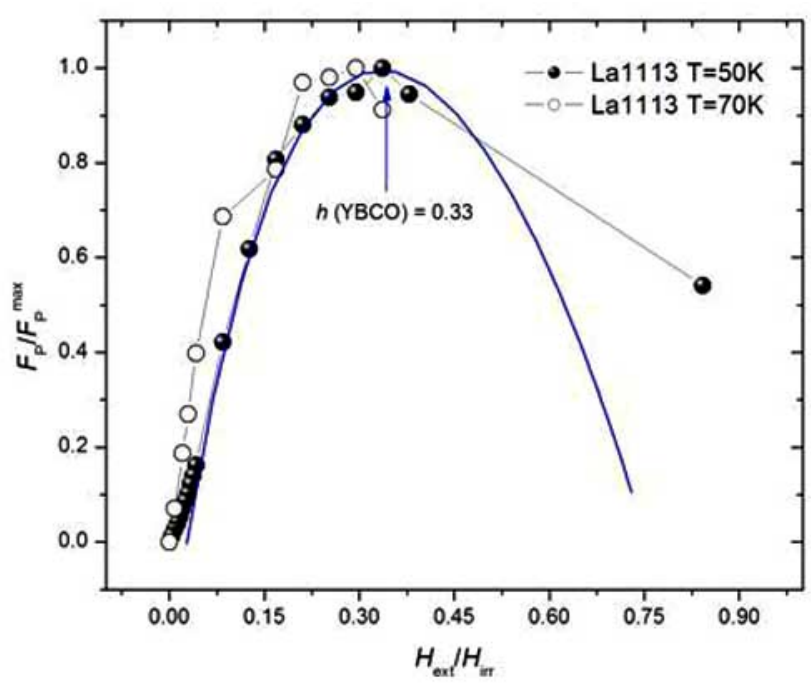

(b)

Fig. (4). Field dependence of $J_{\mathrm{C}}$ obtained from magnetization measurements (a) and normalized pinning force (b) for bulk $\mathrm{LaBaCaCu}_{3} \mathrm{O}_{7}$ at $50 \mathrm{~K}$ and $70 \mathrm{~K}$. 
Fig. (5) shows the DC susceptibility $(\chi)$ versus temperature of $29.5 \mathrm{mg}$ of La1113 in the normal state under $5 \mathrm{kOe}$ applied field. $\chi^{-1}(T)$ is also shown in the figure. From the plot, the susceptibility is positive and follows the typical Curie-Weiss law:

$$
\chi=\chi_{0}+\frac{C}{T-\theta_{p}}
$$

Where $\chi_{0}=1.6 \times 10^{-4} \mathrm{emu} / \mathrm{Oe}-\mathrm{mol}$ is the temperature independent susceptibility, $C=0.207 \mathrm{emu}-\mathrm{K} / \mathrm{Oe}-\mathrm{mol}$ is the Curie constant which is related to the effective paramagnetic moment of the ions ( $\left.\mu_{\mathrm{eff}}\right)$.

$\theta_{\mathrm{P}}$ in equation (3) is the Curie-Weiss temperature and after fitting the plot a value of $-98.4857 \mathrm{~K}$ has been estimated. The negative value of $\theta_{\mathrm{P}}$ indicates antiferromagnetic coupling between the ions in the sample. However, since La, $\mathrm{Ca}$ and $\mathrm{Ba}$ do not have a magnetic moment, decoupling should be between the $\mathrm{CuO}_{2}$ superconducting planes and the $\mathrm{Cu}-\mathrm{O}$ chains in the structure. Note that $\theta_{\mathrm{P}}$ for $\mathrm{YBCO}$ is -20 , and removal of oxygen from its structure lowers the CurieWeiss temperature as it has been demonstrated by Tarascon and co-workers [38]. This suggests that the lower $\theta_{\mathrm{P}}$ value of La1113 might be related with the oxygen deficiency in its structure.

The total independent temperature $\chi_{0}$ in equation (3) is given by:

$\chi_{0}=\chi_{\text {core }}+\chi_{\text {Pauli }}+\chi_{\text {Landau }}$

Where $\chi_{\text {core }}$ is the core diamagnetism term, $\chi_{\text {Pauli }}$ is the Pauli paramagnetism arising from the conduction electrons, and $\chi_{\text {Landau }}$ is the diamagnetic orbital contribution due to the conduction electrons. At first approximation (i.e. for the free-electron model) [39]:

$\chi_{\text {Landau }}=-\frac{1}{3} \chi_{\text {Pauli }}$

Then the equation (4) is reduced to: $\chi_{\text {Pauli }}=\frac{3}{2}\left(\chi_{0}-\chi_{\text {core }}\right)$

The core diamagnetisms for all La1113 atoms are given in Table 1 [40]. They give a core susceptibility of $-1.77 \times 10^{-4}$ $\mathrm{emu} / \mathrm{Oe}$-mol for $\mathrm{LaCaBaCu}_{3} \mathrm{O}_{7}$. Using this value in the last equation, the $\chi_{\text {Pauli }}$ obtained is $5.1 \times 10^{-4} \mathrm{emu} / \mathrm{Oe}-\mathrm{mol}$.

Table 1. Tabulated Values of the Core Diamagnetism for the $\mathrm{LaCaBaCu}_{3} \mathrm{O}_{7}$ Atoms [40]

\begin{tabular}{|c|c|}
\hline Atom & $\chi_{\text {core }}\left(\mathbf{x 1 0} \mathbf{~}^{6} \mathbf{e m u} / \mathbf{O e}-\mathbf{m o l}\right)$ \\
\hline \hline $\mathrm{La}^{3+}$ & 20 \\
\hline $\mathrm{Ba}^{2+}$ & -32 \\
\hline $\mathrm{Ca}^{2+}$ & -8 \\
\hline $\mathrm{Cu}^{2+}$ & -11 \\
\hline $\mathrm{O}^{2+}$ & -12 \\
\hline $\mathrm{LaCaBaCu}_{3} \mathrm{O}_{7}$ & $\chi_{\text {core }}=1.77 \times 10^{-4} \mathrm{emu} / \mathrm{Oe}-\mathrm{mol}$ \\
\hline
\end{tabular}

Utilizing the known Pauli susceptibility obtained above, the value of the specific heat capacity, $\gamma$, can be estimated using the free-electron expression:

$\gamma=\frac{1}{3}\left(\frac{\pi k_{B}}{\mu_{B}}\right)^{2} \chi_{\text {Pauli }}$

Where $k_{\mathrm{B}}$ is the Boltzmann constant $\left(1.3806504 \times 10^{-23} \mathrm{~J}-\mathrm{K}^{-1}\right)$ and $\mu_{\mathrm{B}}$ is the Bohr magneton $\left(9.274009 \times 10^{-24} \mathrm{~J}^{-\mathrm{T}^{-1}}\right)$. Thus, $\gamma$ for $\mathrm{LaCaBaCu}_{3} \mathrm{O}_{7}$ is $37.2 \mathrm{~mJ} / \mathrm{K}^{2}$ mol. This value is different from that obtained by Peng and co-workers for La1113 $\gamma=48.1 \mathrm{~mJ} / \mathrm{K}^{2}$ mol [14] when applying $30 \mathrm{kOe}$ external magnetic field and fitting the susceptibility with two linear functions.

The temperature dependence of the magnetic moment in the superconducting state has been measured under different external magnetic fields. Fig. (6a) shows one of these measurements at $\mathrm{ZFC}$ and $\mathrm{FC}$ at $H_{\mathrm{ext}}=5 \mathrm{Oe}$. According to the

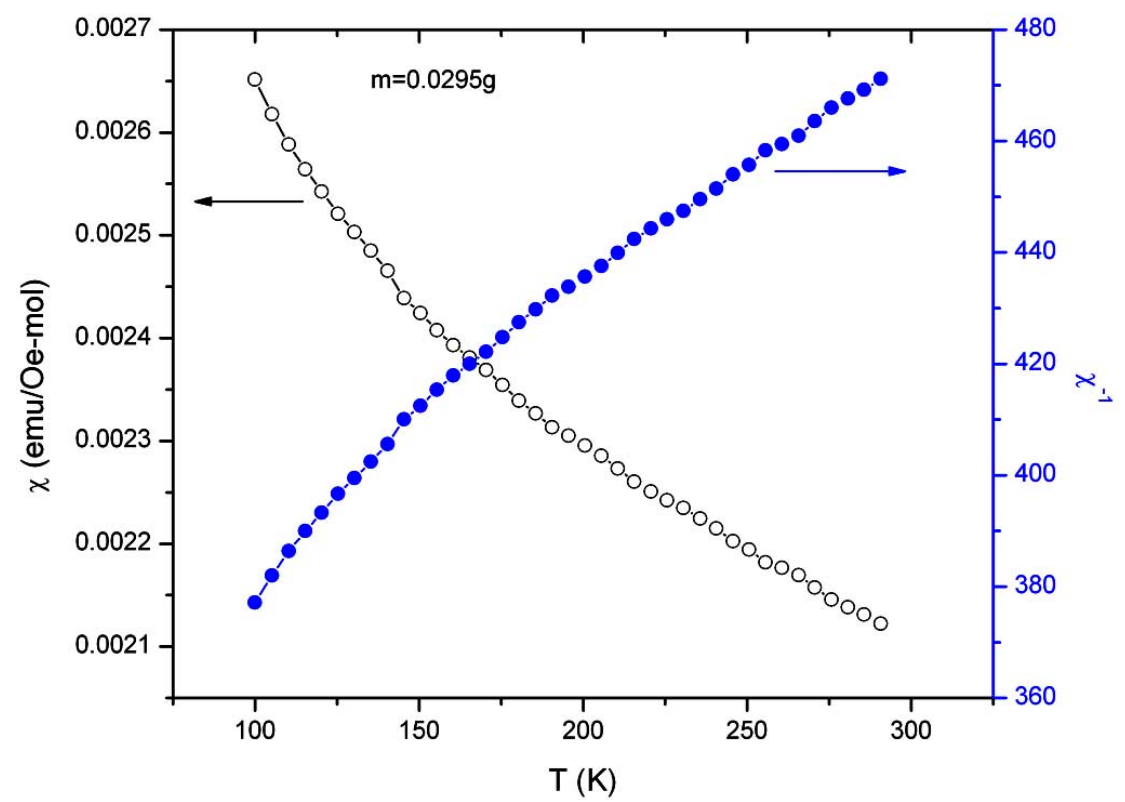

Fig. (5). Normal state DC-susceptibility versus temperature of $\mathrm{LaBaCaCu}_{3} \mathrm{O}_{7}$ under $5 \mathrm{kOe}$ external field. 


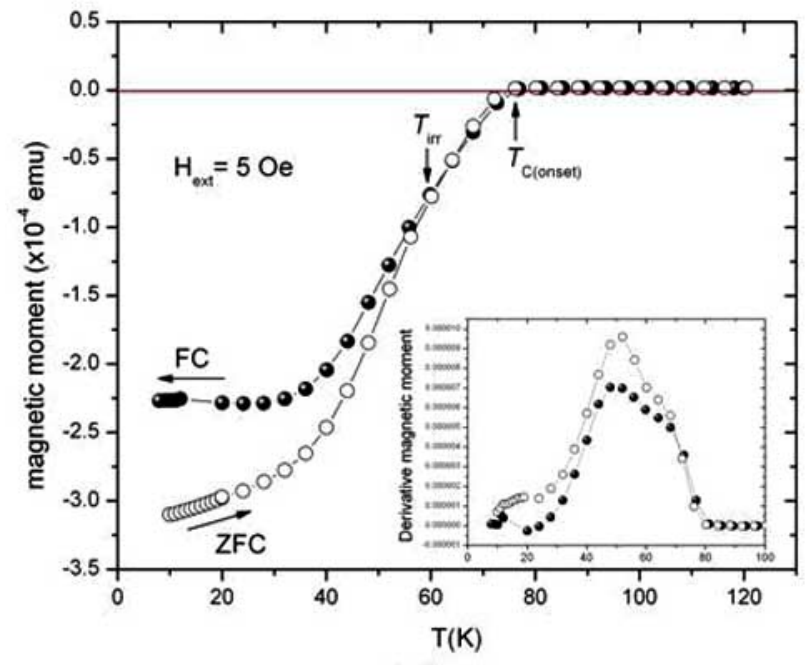

(a)

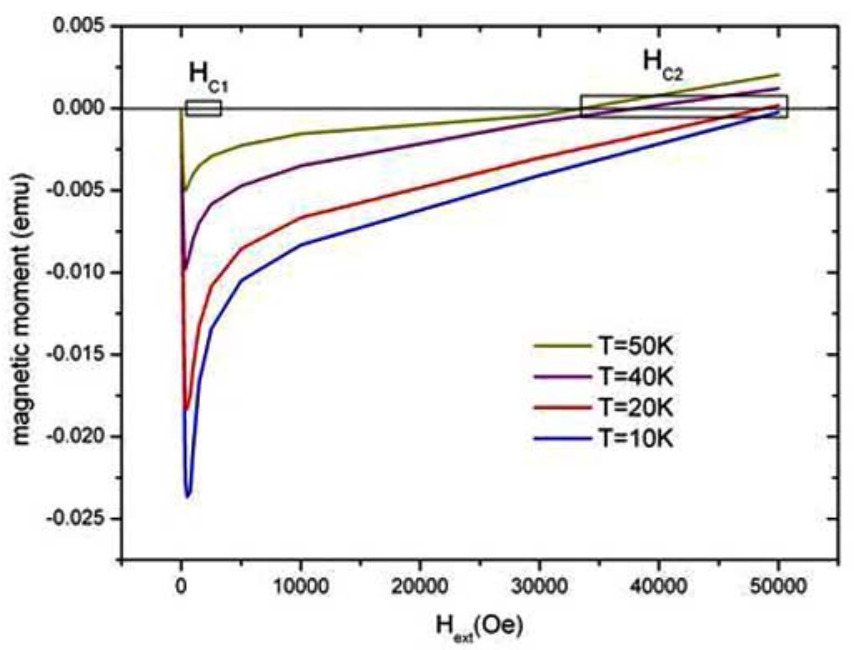

(b)

Fig. (6). Magnetic response of the $\mathrm{LaCaBaCu}_{3} \mathrm{O}_{7}$ as (a) a function of temperature under an external 5 Oe magnetic field (the arrows indicate $T_{\text {irr }}$ and $\mathrm{T}_{\mathrm{C}(\text { onset })}$ ) (b) function of the applied magnetic field for four different temperatures below $T_{\mathrm{C}}$.

figure La1113 becomes diamagnetic from the onset temperature $78 \mathrm{~K}$ and the diamagnetic saturation is reached at low temperatures. The transition widths for the diamagnetic saturation are better seen by taking the derivative of the magnetization plots. As shown in the inset plot, a wide transition width of around $40 \mathrm{~K}$ is observed. This behavior is a characteristic of all bulk superconductors caused mainly by its granular nature together with possible secondary phases and thus the grain boundaries could be considered then as weak Josephson junctions [9]. The irreversibility temperature $T_{\text {irr }}$ (temperature which separates reversible and irreversible regions) is determined in this paper from the points in which ZFC and FC $M(T)$ loops separate. Thus under an external applied magnetic field of $5 \mathrm{Oe}$ as in the figure, $T_{\text {irr }}=60 \mathrm{~K}$. In general, multiple $T_{\text {irr }}$ obtained from different $M(\mathrm{~T})$ measurements define the irreversibility line in a magnetic diagram phase as will be discussed next. It is also possible to derive the magnetic moment behavior as a function of different applied magnetic fields from multiple $M(\mathrm{~T})$ measurements, as shown in Fig. (6b), in which the plot is derived for four different temperatures below $T_{\mathrm{C}}$. In the plot, the peaks indicate the lower critical field $H_{\mathrm{C} 1}$, and the upper critical field $H_{\mathrm{C} 2}$ can be obtained from the intercepts with the abscissa [39]. Similar to the direct-measured hysteresis loops discussed above, both critical fields tend to be high as temperature approaches $0 \mathrm{~K}$ and they decrease continuously with increasing temperature until the transition temperature $T_{\mathrm{C}}$ where they become zero. Thus it is expected that $M(\mathrm{H})$ loops in both situations contracts as temperature increases.

From the collected data and plots described above, we derived a magnetic phase diagram for the La1113 which is presented in Fig. (7). As long as the external flux density

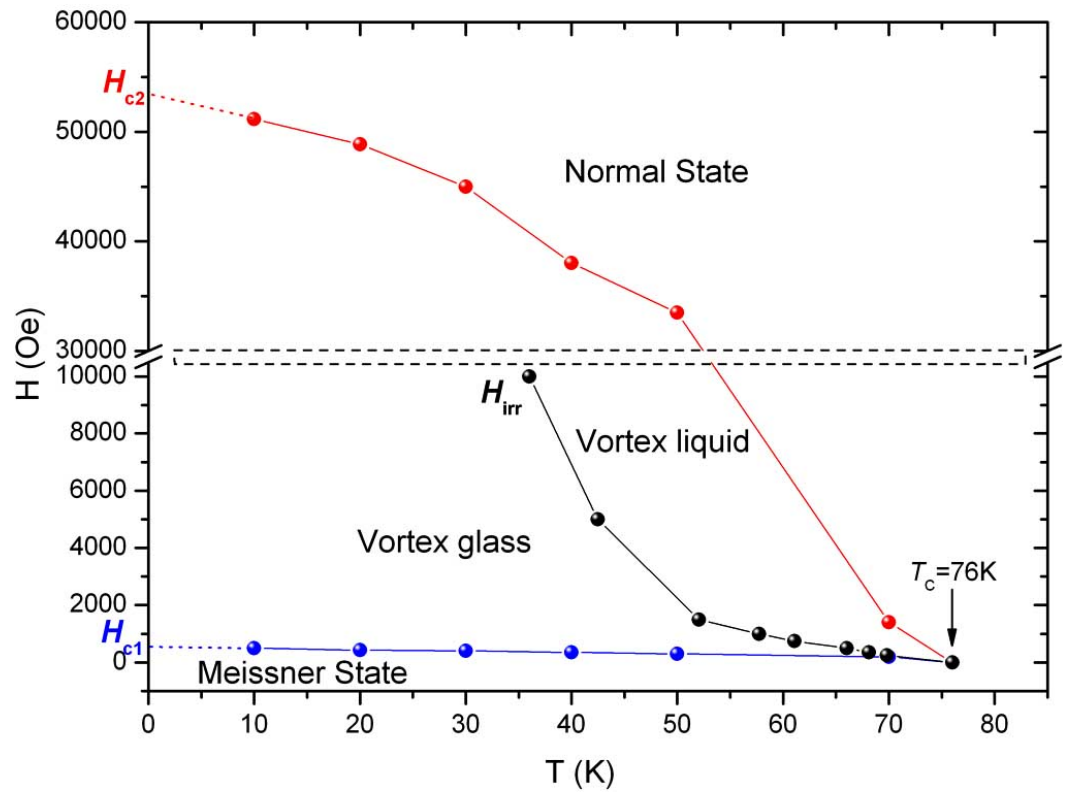

Fig. (7). Magnetic phase diagram of $\mathrm{LaCaBaCu}_{3} \mathrm{O}_{7}$. 
does not exceed $H_{\mathrm{Cl}}$, the bulk La1113 is in a diamagnetic Meissner state, where the magnetic flux is completely expelled from the interior by surface currents. In the area between $H_{\mathrm{C} 1}(T)$ and $H_{\mathrm{C} 2}(T)$, the magnetic flux penetrates the bulk Lal113 in the form of flux lines (mixed state). Magnetic flux motion has to be prevented by flux pinning, i.e the magnitude of $F_{\mathrm{P}}$. As long as the flux lines are pinned, the maximum supercurrent density $J_{\mathrm{C}}$ can flow without any loss. The critical current density $J_{\mathrm{C}}$ depends on the applied magnetic field and on the temperature. If the current density exceeds $J_{\mathrm{C}}\left(H_{\mathrm{irr}}<H<H_{\mathrm{C} 2}\right)$, then the flux line lattice (or parts of it) starts to move. Because of thermal fluctuations, the mobility of the flux line lattice strongly increases with applied magnetic field and temperature. Above the irreversible field $H_{\text {irr }}$ the vortex lattice becomes so strong that currents cannot flow without losses although the superconductor La1113 is not yet in the normal state. This means that possible applications of La1113 are restricted to the field range below the $H_{\text {irr. }}$. At first approximation, $H_{\text {irr }}$ scales with the applied field following a trend:

$$
H_{i r r}=H_{i r(0)}\left(1-\frac{T}{T_{C}}\right)^{n}
$$

Where $H_{\mathrm{ir}(0)}=48 \mathrm{kOe}, T_{\mathrm{C}}=76 \mathrm{~K}$ and the exponent $n$ provides an indication for applications of the superconductor (it varies considerably in different HTCS [41-44]). The shape of the irreversibility field line obtained here is quite similar to that obtained for bulk YBCO in the range 55K-90K [9]. The exponent $n$ in the temperature range $41 \mathrm{~K}-76 \mathrm{~K}$ is 2.8 , this value is double than that obtained for bulk YBCO $(n \approx 1.4)$. Interestingly, Küpfer and co-workers [45] have found that after reducing the oxygen content in the structure of YBCO, $H_{\text {irr }}$ shifts to lower values. Although La1113 has a structure with deficiency of oxygen in the $\mathrm{Cu}-\mathrm{O}$ chains and the shape of the irreversibility line similar to YBCO, we can not assure that $n$ and the low irreversibility line are exclusively originated from the arrangement of oxygen atoms in the structure, because the grain boundaries and secondary phases might contribute in this property as well. Finally, extrapolating $H_{\mathrm{C} 2}$ and $H_{\mathrm{C} 1}$ in the figure to $T=0$, values of $H_{\mathrm{C} 2(0)} \approx 53.5 \mathrm{kOe}$ and $H_{\mathrm{C} 1(0)} \approx 0.5 \mathrm{kOe}$ can be estimated.

\section{CONCLUSION}

Despite the fact that some similarities between $\mathrm{LaCaBaCu}_{3} \mathrm{O}_{7}$ and $\mathrm{YBCO}$ structures do exist, there are also remarkable differences in their magnetic behaviour. Below the $T_{\mathrm{C}}$, the magnetic flux can penetrate easily the La1113 in its vortex region $\left(H_{\mathrm{C} 1}<H<H_{\mathrm{C} 2}\right)$ and fewer fields can be trapped than in YBCO. The critical current density for La1113 is determined by the typical overall connectivity of the polycrystal in which current flows through inter-grains, and a peak effect probably caused by vortex pinning in its structure or by the presence of the secondary phases. The specific heat coefficient of La1113 $\left(37.2 \mathrm{~mJ} / \mathrm{K}^{2} \mathrm{~mol}\right)$ is lower than that for YBCO $\left(54 \mathrm{~mJ} / \mathrm{K}^{2} \mathrm{~mol}[46]\right)$. In the temperature range $41 \mathrm{~K}-76 \mathrm{~K}$, the irreversible line in the magnetic phase diagram of the bulk La1113 has a similar trend than that for YBCO. However, the fitted-exponent $n$ of this trend (2.8) is double than for bulk YBCO $(n \approx 1.4)$, suggesting that the bulk
La1113 can support lower applied magnetic fields than YBCO.

\section{ACKNOWLEDGEMENTS}

This work is supported by the European Union Programme ALBAN (grant No E06D101257PE) and the Cambridge Overseas Trust. The work in Japan is supported by the Japan Society for the Promotion of Science (grant No. PE10027). A. Bustamante, J. Feijoo and A. Osorio thank financial support from the Consejo Superior de Investigaciones (University of San Marcos).

\section{REFERENCES}

[1] Narlikar AV, Narasimha CVR, Agarwal SK. Substitutional studies on high temperature superconductors. In: Narlikar A, Ed. Studies of High Temperature Supercondcutors, Advances in research and applications. USA: Nova Science Publishers 1989; vol. 1.

[2] Karen P, Fjellvag H, Kjekshus A, Andresen AF. Chemical pressure and other effects of strontium substitution in $\mathrm{YBa}_{2} \mathrm{Cu}_{3} \mathrm{O}_{9-\delta}$. Solid State Chem 1991; 92: 57-67.

[3] Cava RJ. Oxide superconductors. J Am Ceram Soc 2000; 83(1): 528.

[4] Daeumling M, Seuntjens JM Larblestier DC. Oxygen-defect flux pinning, anomalous magnetization and intra-grain granularity in $\mathrm{YBa}_{2} \mathrm{Cu}_{3} 0_{7-\delta}$. Nature 1990; 346: 332 .

[5] Weber HW. Flux Pinning. In: Gscheidner KA, Jr, Eyring I, Maple MB, Eds. Hanbook on the physics and chemistry of rare earths. Amsterdan: Elsevier 1999.

[6] Oussena M, de Groot PAJ, Gagnon R, Taillefer I. Lock-in oscillations in magnetic hysteresis curves of $\mathrm{YBa}_{2} \mathrm{Cu}_{3} \mathrm{O}_{7-\mathrm{x}}$ single crystals. Phys Rev Lett 1994; 72: 3606.

[7] Erb A, Walker E, Genoud JY, Flükiger R. Reversible suppression of the so-called fishtail effect in ultra pure single crystals of YBa2Cu3O7- $\delta$. Physica C 1997; (Part 4), 282-287: 2145-46.

[8] Erb A, Manuel AA, Dhalle M, et al. Experimental evidence for fast cluster formation of chain oxygen vacancies in $\mathrm{YBa} 2 \mathrm{Cu} 3 \mathrm{O} 7-\delta$ as the origin of the fishtail anomaly. Solid State Commun 1999; 112: 245.

[9] Gernot K, Günter F, Wolf-Rüdiger C, Hardo M, Ryszard P. High temperature supercondcutors bulk materials. Weinheim: WileyVCH Verlag GmbH\&Co. KGaA 2006.

[10] Gunasekaran RA, Yakhmi JV, Iyer RM. On the evolution of superconductivity in $\mathrm{La}_{1.5-\mathrm{x}} \mathrm{Ba}_{1.5-\mathrm{x}} \mathrm{Ca} 2_{\mathrm{x}} \mathrm{Cu}_{3} \mathrm{O}_{7-\delta}$. Physica C 1993; 208: 143 .

[11] De Leeuw DM, Mutsaers CAHA, van Hall HAM, Verweij H, Carim AH, Smoorenberg HCA. Properties of the tetragonal superconductor $\mathrm{CaBaLaCu}_{3} \mathrm{O}_{\mathrm{x}}$. Physica C 1988; 156: 126 .

[12] Yagi T, Domon M, Okajima Y, Yamaya K. Effect of oxygen deficiency on the normal and superconducting properties of $\mathrm{CaLaBaCu}_{3} \mathrm{O}_{\mathrm{y}}$. Physica C 1991; 173: 453.

[13] Gunasekaran RA, Gopalakrishnan IK, Sastry PV, Yakhmi JV, Iyer RM. Phase stability and superconducting characteristics of $\mathrm{CaBa}$ $\left(\mathrm{La}_{1-\mathrm{x}} \mathrm{R}_{\mathrm{x}}\right) \mathrm{Cu}_{3} \mathrm{O}_{7-\delta}(\mathrm{R}=$ rare earth $)$ system. Physica C 1992; 199: 240.

[14] Peng JL, Klavis P, Shelton RN, et al. Preparation, characterization, and superconducting properties of tetragonal $\mathrm{LaBaCaCu}_{3} \mathrm{O}_{7+\delta}$. Phys Rev B 1989; 39: 9074.

[15] Fu WT, Zand Bergen HW, Van Der Beek CJ, De Jongh LJ. Hightemperature superconductivity in $\mathrm{LaBaCaCu}_{3} \mathrm{O}_{6.85}$. Physica $\mathrm{C} 1998$; 156: 133

[16] Carim AH, de Jong AF, de Leeuw DM. Ordering phenomena in the tetragonal superconductor $\mathrm{CaBaLaCu}_{3} \mathrm{O}_{7-\delta}$. Phys Rev B 1988; 38: 7009.

[17] Yagi T, Domon M, Okajima Y, Yamaya K. Effect of oxygen deficiency on the normal and superconducting properties of $\mathrm{CaLaBaCu}_{3} \mathrm{O}_{\mathrm{y}}$. Physica C 1991; 173: 453.

[18] Yamaya K, Yagi T, Okajima Y. Transport properties near superconducting-nonsuperconducting phase boundary in $\mathrm{CaLaBaCu}_{3} \mathrm{O}_{\mathrm{y}}$. Solid State Commun 1993; 87: 1113 .

[19] Awana VPS, Narlokar AV. Physical characterization of pure and substituted tetragonal $\mathrm{LaBaCaCu}_{3} \mathrm{O}_{7-\delta}$ superconductor. Mod Phys Lett B 2001; 15(14): 415-53. 
[20] Rajvir S. Superconductivity in $\mathrm{Zn}$-doped tetragonal $\mathrm{LaBaCaCu}_{3} \mathrm{O}_{7-\delta}$ systems. Phys Rev B 1997; 55(2): 1216-22.

[21] De Los Santos LV, Bustamante AD, Flores JS, Gonzalez JG. Preparation and characterization of the superconductor $\mathrm{CaLaBaCu}_{2.8}\left(\mathrm{PO}_{4}\right)_{0.2} \mathrm{O}_{6.2}$ compound. Physica C 2004; 408-10: 44-5.

[22] De Los Santos LV, Bustamante AD, Gonzalez JG, Flores JS, Obradors $\mathrm{X}$. Superconductivity in the System $\mathrm{CaLaBaCu}_{3-}$ $\mathrm{x}\left(\mathrm{PO}_{4}\right)_{\mathrm{X}} \mathrm{O}_{7-\delta}$ with $\mathrm{X}=0.1,0.3,0.5$. J Phys Chem Solids 2006; 67: 605-9.

[23] Bustamante AD, Bellido RQ, De Los Santos LV, González JG. Preparation and characterization of the superconductor $\mathrm{CaLaBaCu}_{2.8}\left(\mathrm{BO}_{3}\right)_{0.2} \mathrm{O}_{6.4}$ compound. Physica C 2004; 408-410: 884-5.

[24] Feijoo JAL, Osorio AM, Bustamante AD, et al. Caracterización del cerámico superconductor $\mathrm{CaLaBaCu}_{3} \mathrm{O}_{7-\delta}$ obtenido por el método sol gel. Rev Soc Quim Peru 2007; 73(4): 208-14.

[25] González JC, De Los Santos LV, Feijoo J, Osorio A, Bustamante AD. Difracción de electrones del compuesto $\mathrm{CaLaBaCu}_{3} \mathrm{O}_{7-\delta}$. Rev Soc Quim Peru 2009; 75(4): 422-30.

[26] Bustamante AD, Osorio AA, De Los Santos LV, et al. Synthesis of $\mathrm{YBa}_{2} \mathrm{Cu}_{3} \mathrm{O}_{7-\delta}$ using oxalate precursor and sol-gel method. Adv Sci Technol 2006; 47: 37-42.

[27] Dennis CB. Elements of X-ray diffraction. 3rd ed. Upper Saddle rive, N.J. Prentice Hall, London, Prentice-Hall International 2001.

[28] Koblischka MR, van Dalen AJJ, Higuchi T, Sawada K, Yoo SI, Murakami M. Observation of multiple peaks in the magnetization curves of $\mathrm{NdBa}_{2} \mathrm{Cu}_{3} \mathrm{O}_{7}$ single crystals. Phys Rev B 1996; 54: R6893.

[29] Krabbes G, Fuchs G, Schätzle $\mathrm{P}$, et al. $\mathrm{Zn}$ doping of $\mathrm{YBa}_{2} \mathrm{Cu}_{3} \mathrm{O}_{7}$ in melt textured materials: peak effect and high trapped fields. Physica C 2000; 330: 181.

[30] Bean CP. Magnetization of high-field superconductors. Rev Mod Phys 1964; 36: 31 .

[31] Jin S, Sherwood RC, Gyorgy EM, et al. Large magnetic hysteresis in a melt-textured $\mathrm{Y}-\mathrm{Ba}-\mathrm{Cu}-\mathrm{O}$ superconductor. Appl Phys Lett 1989; 54(6): 584-6.

[32] Fuchs G, Gladun A, Fischer K, Rodig C. Critical current density in Ag-sheathed wires of YBaCuO. Cryogenics 1992; 32: 591.
[33] Peterson RI, Ekin JW. Critical-current diffraction patterns of grainboundary Josephson weak links. Phys Rev B 1990; 42: 8014.

[34] Däumling M, Seuntjens JM, Larbalestier DC. Oxygen-defect flux pinning, anomalous magnetization and intra-grain granularity in $\mathrm{YBa}_{2} \mathrm{Cu}_{3} 0_{7-\delta}$. Nature 1990; 346: 332.

[35] Koblischka MR. Pinning forces and scaling in high- $\mathrm{T}_{\mathrm{c}}$ superconductors. Physica C 1997;282-287: 2193-4.

[36] Koblischka MR, Murakami M. Pinning mechanisms in bulk highTc superconductors. Supercond Sci Technol 2000; 13: 738-44.

[37] Blatter G, Feigel'man MV, Geshkenbein VB, Larkin AI, Vinokur VM. Vortices in high-temperature superconductors. Rev Mod Phys 1994; 66: 1125.

[38] Tarascon JM, Mckinnon WR, Greence LH, Hull GW, Vogel EM. Oxygen and rare-earth doping of the 90-K superconducting perovskite $\mathrm{YBa}_{2} \mathrm{Cu}_{3} \mathrm{O}_{7-\mathrm{x}}$. Phys Rev B 1987; 36: 226 .

[39] Poole CP, Farach HA, Creswick RJ, Prozorov R. Superconductivity. $2^{\text {nd }}$ ed. Amsterdam: Academic Press 2007.

[40] White RM. Quantum Theory of Magnetism, $3^{\text {rd }}$ ed, Berlin: Springer 2006.

[41] Müller KA, Takashige M, Bednorz JG. Flux trapping and superconductive glass state in $\mathrm{La}_{2} \mathrm{CuO}_{4-\mathrm{y}}: \mathrm{Ba}$. Phys Rev Lett 1987; 58: 1143 .

[42] Yeshurun Y, Malozemoff AP. Giant flux creep and irreversibility in an Y-Ba-Cu-O crystal: An alternative to the superconductingglass model. Phys Rev Lett 1988; 60: 2202.

[43] Felner I, Wolfus Y, Hilscher G, Pillmayr N. Susceptibility, crystalstructure, and specific-heat studies of the high-Tc superconductor and quenched $\mathrm{YBa}_{2}\left(\mathrm{Cu}_{0.91} \mathrm{Fe}_{0.09}\right)_{3} \mathrm{O}_{\mathrm{x}}$. Phys Rev B 1989; 39: 225.

[44] Yeshurun Y, Felner I, Sompolinsky H. Magnetic properties of a high-Tc superconductor $\mathrm{YBa}_{2} \mathrm{Cu}_{3} \mathrm{O}_{7}$ : Reentry-like features, paramagnetism, and glassy behavior. Phys Rev B 1987; 36: 840 .

[45] Küpfer H, Wolf Th, Meier-Hirmer R, Zhukov AA. Peak effect and vortex phase diagram in twin-free $\mathrm{YBa}_{2} \mathrm{Cu}_{3} \mathrm{O}_{7-\delta}$ single crystals. Physica C 2000; 332: 80.

[46] Nevitt MV, Crabtree GW, Klippert TE. Heat capacity of $\mathrm{YBa}_{2} \mathrm{Cu}_{3} \mathrm{O}_{7-\delta}$ at the superconducting transition temperature. Phys Rev B 1987; 36: 2398 .

Received: February 03, 2010

Revised: April 11, 2010

Accepted: May 10, 2010

(C) De Los Santos et al.; Licensee Bentham Open.

This is an open access article licensed under the terms of the Creative Commons Attribution Non-Commercial License (http://creativecommons.org/ licenses/by-nc/3.0/), which permits unrestricted, non-commercial use, distribution and reproduction in any medium, provided the work is properly cited. 